\section{The Hydrogen Chromosphere}

THE slow decrease of the density of hydrogen with increasing height in the solar atmosphere requires a consideration of the force opposing gravity. As is well known, radiation pressure is insufficient for this purpose, at least if the radiation-intensity is not wholly different from the value derived by extrapolation from the solar spectrum in the visible region.

However, the existence of an outward moving stream of $\mathrm{Ca}^{+}$atoms requires the investigation of the acceleration imparted to the $\mathrm{H}$-atoms. This acceleration depends on the density $\rho$ of the $\mathrm{Ca}^{+}$atoms and their systematic outward velocity $v$. The value of the required product $\rho v$ may be deduced from the contour-asymmetry of the $K$-line, as measured by Minnaert. I have derived the value 1

$$
\rho v=0.8 \times 10^{-12} ;
$$

however, owing to the circumstances of the problem, this value has a large degree of uncertainty and requires revision in the future.

Combining the determination of $\rho v$ with a classical computation ${ }^{2}$ of the effect of the collisions on the hydrogen atoms, I have deduced the ratio of the acceleration imparted to a hydrogen ion at rest to the acceleration of gravity. The resulting value of this ratio is $1 \cdot 1$. The value for a hydrogen atom would be much smaller on account of the shielding effected by the bound electron.

Though inconclusive at present on account of uncertainties in the values involved, this computation induces me to adopt as a working hypothesis the convection of the hydrogen atoms by the outward moving $\mathrm{Ca}^{+}$atoms.

The fact that the motive force of the $\mathrm{Ca}^{+}$ions only is present at comparatively great heights in the solar atmosphere concentrates its action especially on hydrogen.

A more detailed analysis of the motion of hydrogen will be published elsewhere. J. WoLtJeR, $J_{\mathrm{R}}$.

Brêloftpark 28,

Noordwijk, Holland.

$$
\text { ? } \stackrel{\text { B.A.N.N., } 182 .}{\text { B.A.N., } 213 .}
$$

\section{Polarisation of Raman Scattering}

Tre use of an improved technique has enabled us to obtain more precise measurements than formerly of the polarisation of the lines in Raman spectra. A systematic study has now been made with many liquids. An important result obtained is that no line has a depolarisation greater than $6 / 7$, and that earlier reports by various workers indicating a larger depolarisation in certain cases are not correct. The limiting value of $6 / 7$ is obtained in many instances, especially in all cases of a rotational scattering, whether by gases or by liquids. Many vibration lines also exhibit this high degree of depolarisation, as, for example, the frequeneies 605,1584 , and 1605 of benzene, the two bands at 189 and 1398 in formic acid, the line at 750 in thiophene, the frequencies 106, 131, and 403 in tin tetrachloride, and the corresponding lines given by the tetrachlorides of carbon, silicon, and titanium. In all these cases, the explanation suggested in the last paragraph of Bhagavantam's note in Nature of Jan. 30, 1932 (p. 167), is evidently applicable.

A careful determination has been made with the intense 992 line of benzene and it is found that it exhibits a genuine depolarisation of 7 per cent. The tetrachlorides of carbon, silicon, titanium, and tin exhibit an interesting progression in the behaviour of their strongest lines : the lines $459,426,382$, and 367 given respectively by these compounds exhibiting depolarisations of $4,11,12$, and 16 per cent. That these lines, which correspond to a symmetric oscilla. tion of the tetrahedral molecules, exhibit a genuine depolarisation is not surprising in view of the wellattested optical anisotropy of these molecules. It is found that the Rayleigh scattering by all the tetrachlorides is accompanied by a rotational Raman scattering and is depolarised to an appreciable extent, which increases with increasing atomic number of the central atom.

S. Bhagavantam.

210 Bowbazar Street,

Calcutta, India, Feb. 28.

\section{Constitution of the Keratin Molecule}

Is a note which appeared recently in these columns, Speakman and Hirst ${ }^{1}$ recorded comparisons of the work necessary to stretch wool fibres in water, in acids, and after treatment with nitrous acid. The conclusion they reached was that acids disrupt a linkage of the type $\mathrm{R}_{1}-\mathrm{COO}-\mathrm{NH}_{3}-\mathrm{R}_{2}$ in the keratin molecule formed from acidic and basic units in adjacent polypeptide chains. Taking the figures quoted by Marston 2 for the amino-acid composition of wool, it was shown that the dibasic aspartic and glutamic acids were collectively present in quantity roughly proportional to that required on the assumption that they entered into a linkage of the type postulated with the $\epsilon$-amino group of lysine and the guanidine group of arginine.

The suggestion put forward by Speakman and Hirst is worthy of consideration in explaining the properties of wool fibres, although they have neglected in their calculations one factor which makes the agreement or correspondence between the quantities not so close as would appear from their figures. They have assumed that every molecule of glutamic or aspartic acid has one carboxyl group free to enter into salt formation with an amino group. Wool yields upon hydrolysis 1.2 per cent of 'ammonia'- or 'amide'nitrogen. Now, the general opinion among protein chemists is that this ammonia exists in the protein in the form of acid-amide linkages with one or other of the dibasic amino acids. $3,4,5$

Osborne ${ }^{6}$ was one of the first to point out the parallelism between the yields of ammonia and of dicarboxylic acids from various proteins, and although hydroxyglutamic acid was only discovered afterwards, ${ }^{7}$ it will be recalled that Luck ${ }^{8}$ succeeded in isolating a complex from caseinogen, representing at least onethird of its ammonia nitrogen, in which a peptide of lysine glutamic acid amide was the principal constituent. Alternative theories as to the origin of ammonia nitrogen, for example, ureide linkages, ${ }^{9}$ which have at one time or another been proposed, have all had eventually to be abandoned.

Speakman and Hirst must therefore deduct 12.6 per cent by weight of glutamic acid from the 12.9 per cent contained in wool, since this is presumably in combination with the $\mathbf{1} \cdot \mathbf{2}$ per cent of ammonia nitrogen, leaving 0.3 per cent of glutamic acid and 2.3 per cent of aspartic acid (say $2 \cdot 6$ per cent in all) free to enter into the type of union they postulate with the basic amino acids. As they state, "Using Marston's data, the arginine and lysine are together equivalent to an amount of glutamic acid equal to $11 \cdot 4$ per cent by weight of the wool ". Even if the more recent figures of Vickery and Block, 10 independently con. firmed by Stewart and me, ${ }^{11}$ of 8.6 per cent arginine instead of $10 \cdot 2$ per cent and $2 \cdot 3$ per cent lysine instead of 2.8 per cent, be taken, these bases, for complete combination, would require $\mathbf{9 . 5 7}$ per cent of glutamic acid-nearly four times the amount actually available.

So far, no systematic search has been made for 